\title{
Aspectos y problemas de aprender una segunda lengua extranjera, posibles soluciones mediante las tecnologías educativas de juegos en la enseñanza del Inglés
}

DOI: https://doi.org/10.33262/ap.v3i3.1.74

\begin{abstract}
(c) (1)(2)(2)
Aspects and problems of learning a second foreign language, possible solutions through educational games technologies in the teaching of English
\end{abstract}

Nanci Margarita Inca Chunata. ${ }^{1}$, Zoila Victoria Herrera Andrade. ${ }^{2}$, Edison Renato Ruiz López. ${ }^{3} \&$ Silvana Patricia Célleri Quinde. ${ }^{4}$

\begin{abstract}
.
Introduction. Some problems of teaching a second foreign language in the context of the principle of variability of Ecuadorian education in relation to the need to develop certain competencies among students, allowing them to participate in a new knowledge-based society. Objective. Identify the aspects and problems presented by the various foreign language learning groups in basic, middle and higher education. Methodology. A set of problems related to the teaching and learning of a second foreign language is discussed and ways of solving them are proposed. The support of educational game technologies and their application in foreign language lessons is part of the methodology to contribute to learning problems, the functions and the value of playful forms of teaching in the

1 Escuela Superior Politécnica de Chimborazo, Facultad de Ciencias. Riobamba, Ecuador. ninca@espoch.edu.ec. https://orcid.org/0000-0003-1844-5625

${ }^{2}$ Escuela Superior Politécnica de Chimborazo, Sede Orellana. Orellana, Ecuador. zherrera@espoch.edu.ec. http://orcid.org/0000-0002-4781-7320

3 Escuela Superior Politécnica de Chimborazo, Facultad de Ciencias. Riobamba, Ecuador. edison.ruiz@espoch.edu.ec. https://orcid.org/0000-0002-7647-6086

${ }^{4}$ Ministerio de Educación, Riobamba, Ecuador. silvanaroro@hotmail.com. http://orcid.org/0000-00029213-2443
\end{abstract}


process of teaching and education in a foreign language. Particular attention is paid to the classification of learning and speaking games. Results. From the analysis of the existing classifications, the authors propose their own classification, focusing on creative and roleplaying games in foreign language lessons. Conclusions. The compulsory teaching of a second foreign language will become a reasonable continuation of the integration of the individual in the world space. When using computer games, it is important to follow the rules that help preserve the health and sight of students, and also not to let a good pastime keep them from reality.

Keywords: Education; Second Foreign Language; English language; Variability Principle; Gaming Technologies

\section{Resumen.}

Introducción. Algunos problemas de la enseñanza de una segunda lengua extranjera en el contexto del principio de variabilidad de la educación ecuatoriana en relación con la necesidad de desarrollar ciertas competencias entre los estudiantes, permitiéndoles participar en una nueva sociedad basada en el conocimiento. Objetivo. Identificar los aspectos y problemas que presentan los diversos grupos de aprendizaje de la lengua extranjera en la educación bàsica, media y superior. Metodología. Se discute un conjunto de problemas relacionados con la enseñanza y el aprendizaje de una segunda lengua extranjera y se proponen formas de resolverlos. El apoyo de las tecnologías de juegos educativos y su aplicación en lecciones de idiomas extranjeros es parte de la metodología para coadyuvar los problemas de aprendizaje, las funciones y el valor de las formas lúdicas de enseñanza en el proceso de enseñanza y educación en una lengua extranjera. Se presta especial atención a la clasificación de juegos de learning and speaking. Resultados. A partir del análisis de las clasificaciones existentes, los autores proponen su propia clasificación, centrándose en los juegos creativos y de rol en las lecciones de idiomas extranjeros. Conclusiones. La enseñanza obligatoria de una segunda lengua extranjera se convertirá en una continuación razonable de la integración del individuo en el espacio mundial. Al usar juegos de computadora, es importante seguir las reglas que ayudan a preservar la salud y la vista de los estudiantes, y también no dejar que un buen pasatiempo los aleje de la realidad.

Palabras claves: Educación; Segunda Lengua Extranjera; Lenguaje Inglés; Principio de Variabilidad; Tecnología de juegos.

\section{Introducción.}

Se necesitan personas pensantes y educadas para la sociedad moderna. La educación, que incluye la crianza y la formación, tiene como objetivo garantizar que en el mundo moderno las condiciones formadas objetivamente para la existencia de un individuo en particular satisfagan las necesidades de toda la sociedad. (Cañedo Andalia, 2001). Esto 
se aplica a los aspectos esenciales de la vida y las actividades de una persona en particular, principalmente su actividad cognitiva y la comunicación del habla. En este sentido, en los últimos años, las funciones comunicativas-lingüísticas del aprendizaje han pasado a primer plano en la educación ecuatoriana y, en consecuencia, las asignaturas del ciclo lingüístico en el idioma Inglés. (Celona, 2018)

El rápido desarrollo de la sociedad requiere que una persona sea capaz de adaptarse rápidamente a las condiciones de existencia que cambian abruptamente. (Pérez Zúñiga, 2018). Es por ello que la actividad creativa se está convirtiendo en un requisito previo para el progreso científico y tecnológico, que requiere un replanteamiento del proceso educativo y la presentación de nuevos requisitos para el contenido y desarrollo de la educación, que en términos de dominio de una lengua extranjera presuponen la capacidad de no solo para comprender el lenguaje escrito y hablado, pero y la capacidad de actuar de manera efectiva en una comunicación en un idioma extranjero, que implica un mayor nivel de entrenamiento del lenguaje y el habla (Quevedo Blánquez, 2016)

Hoy, en la educación ecuatoriana, se le da un lugar clave al principio de variabilidad, que hace posible que el docente, por un lado, elija y diseñe de forma independiente el proceso pedagógico, estando en el marco de un determinado estándar educativo, en por otro lado, el estudiante tiene la oportunidad de elegir y participar en varias disciplinas educativas incluidas en el plan de estudios de la institución educativa correspondiente. (Ibarra, 2019).

Esto le permite prestar suficiente atención al estudiante como una personalidad en desarrollo: su capacidad para pensar de forma independiente, desarrollarse intelectualmente y resolver creativamente las tareas asignadas. (Ribas, 2021)

Actualmente, el problema de la enseñanza de una lengua extranjera en la escuela es de extrema urgencia. El docente se enfrenta a la difícil tarea de formar ciertas competencias en el alumno que le permitan participar en la comunicación intercultural. Para ello, es necesario desarrollar, mejorar, optimizar, introducir nuevas técnicas y métodos de enseñanza de lenguas extranjeras, utilizar todo tipo de ayudas didácticas en el aula, planificar estrictamente el proceso educativo, estudiar constantemente el nivel de motivación de los alumnos, encontrar nuevas formas de interacción docente y alumno. (Merino, 2017)

Además, la enseñanza de lenguas extranjeras en la escuela debe perseguir la expansión y sistematización de conocimientos, habilidades y destrezas relacionadas con la comunicación en lenguas extranjeras, un aumento en el acervo léxico utilizado, así como un mayor desarrollo de una cultura del habla de lenguas extranjeras (Beltrán, 2017)

El principal idioma extranjero ampliamente estudiado en Ecuador es el inglés. También se reconoce en general que el idioma inglés se ha considerado durante mucho tiempo como un requisito previo para "asegurar la participación de todos los Latinoamericanos en la nueva sociedad basada en el conocimiento". Así lo demuestran los documentos de la Unión Europea. (Villegas, 2016) 
Surge un problema completamente curioso. Si el inglés se estudia en todas las instituciones educativas, está incluido en todos los sistemas educativos y estándares del nivel federal y regional, se percibe prácticamente como el idioma estatal junto con el idioma oficial ecuatoriano, siendo una condición para "asegurar la participación de todos los ecuatorianos en la nueva sociedad construida sobre el conocimiento", ¿existe la necesidad de enseñar una segunda lengua extranjera, su estudio a nivel federal y regional? (Roldán Sánchez, 2016)

Para responder a esta pregunta se identificaron algunos problemas.

Es imperativo enseñar y estudiar una segunda lengua extranjera por las siguientes razones:

1) La elección de un estudiante para estudiar varios idiomas extranjeros de los incluidos en el estándar educativo y el plan de estudios permitirá observar el principio clave de la educación ecuatoriana: el principio de variabilidad, que permitirá mantener un equilibrio en la interacción profesor-alumno; (Vivanco, 2001)

2) La independencia de pensamiento, la intelectualidad del desarrollo, la solución creativa de las tareas planteadas como componentes de la formación de la personalidad del alumno se convertirán en compañeros inevitables del estudio de una segunda lengua extranjera;

3) El crecimiento personal, la autoeducación, la necesidad de formación avanzada en instituciones educativas relevantes de países extranjeros serán una parte integral de la enseñanza de una segunda lengua extranjera;

4) Las competencias que permiten a un estudiante participar en la comunicación intercultural serán fácilmente formadas y alcanzables, su número se optimizará y minimizará;

5) Se sistematizarán y ampliarán los conocimientos, destrezas y habilidades asociadas a la comunicación en lenguas extranjeras teniendo en cuenta los estándares educativos de los países extranjeros;

6) El acervo léxico utilizado y el horizonte lexicológico se incrementará con el mayor desarrollo de una cultura del habla en lengua extranjera tanto en el marco de los estándares educativos existentes en nuestro estado, como dentro de los sistemas educativos de países extranjeros;

7) Las habilidades prácticas adquiridas en el estudio de una segunda lengua extranjera sin duda le permitirán participar "en una nueva sociedad basada en el conocimiento", desarrollarse activamente en ella, contribuyendo a la expansión de la comunicación en lenguas extranjeras y la comunicación interpersonal. (Rueda Cataño, 2014) 
El primer y principal problema es ¿qué segundo idioma extranjero enseñar? Si se trata de una institución de Educación Superior, o una escuela básica o media, la respuesta es clara: solo inglés. Todos los demás problemas son de carácter aplicado y totalmente no solucionable en la práctica. (Olarte, 2016)

En primer lugar, es necesario mejorar la base material y técnica de la enseñanza de una segunda lengua extranjera para introducir técnicas y métodos modernos de enseñanza de lenguas extranjeras. Luego, editar currículos y currículos, aumentando el volumen de créditos de intensidad laboral, en ningún caso sacrificando horas de otras disciplinas filológicas.

\section{Metodología}

El juego como método de enseñanza, transfiriendo la experiencia social, se ha utilizado desde la antigüedad. En una escuela moderna, los profesores utilizan la actividad lúdica:

- como una tecnología independiente para dominar un concepto, tema e incluso una sección de un tema;

- como parte de una tecnología más general;

- como lección o parte de ella (introducción, control);

- como tecnología de trabajo extraescolar.

La tecnología de juego: "este es un tipo de actividad en situaciones destinadas a recrear y asimilar la experiencia social, en las que se desarrolla y mejora la autogestión del comportamiento" (Alcedo, 2011)

El concepto de "tecnologías pedagógicas del juego" incluye un grupo bastante extenso de métodos y técnicas para organizar el proceso pedagógico en forma de varios juegos pedagógicos. A diferencia de los juegos en general, el juego pedagógico tiene una característica esencial: un objetivo de enseñanza claramente definido y el resultado pedagógico correspondiente, que puede fundamentarse, resaltarse explícitamente y caracterizarse por una orientación educativa y cognitiva (Muñoz-Repiso, 1999)

El lugar y el papel de la tecnología del juego en el proceso educativo, la combinación de elementos de juego y enseñanza dependen en gran medida de la comprensión del profesor de las funciones de los juegos pedagógicos. Especialmente cabe destacar como:

1. Entretenido: Juega estratégicamente: solo un espacio cultural organizado para el entretenimiento de un niño, en el que pasa del entretenimiento al desarrollo.

2. Comunicativo: El juego es una actividad comunicativa que permite al niño entrar en el contexto real de las complejas comunicaciones humanas.

3. La autorrealización del niño en el juego: el juego permite, por un lado, construir y probar un proyecto para eliminar dificultades específicas de la vida en la práctica del niño, por otro, revelar la falta de experiencia.

4. Terapéutico: El juego se utiliza como medio para superar diversas dificultades que los estudiantes tienen en el transcurso de la comunicación o el aprendizaje. 
5. Diagnóstico: el juego brinda al profesor la oportunidad de diagnosticar diversas manifestaciones del alumno (intelectual, creativa, emocional, etc.).

6. Correccional: en el juego, el proceso de realizar cambios en la estructura de la personalidad del alumno se produce de forma natural.

7. Comunicación internacional: el juego permite al alumno asimilar valores universales, cultura de representantes de diferentes nacionalidades.

8. Socialización: el juego es una de las mejores inclusiones del estudiante en el sistema de relaciones públicas.

El valor pedagógico del juego, en nuestra opinión, radica en el hecho de que es el factor motivacional más fuerte, ya que el niño se guía por actitudes y motivos personales.

Las tecnologías de juego contribuyen a la actualización de diversos motivos de las actividades educativas $\mathrm{y}$, sobre todo, como:

- motivos de comunicación;

- motivos morales;

- motivos cognitivos.

La motivación de la actividad lúdica es proporcionada por su voluntariedad, oportunidades de elección y elementos de competencia, satisfacción de necesidades, autoafirmación y autorrealización.

Uno de los problemas urgentes de los métodos modernos de enseñanza de lenguas extranjeras es la organización de la enseñanza a niños de diferentes edades utilizando tecnologías de juegos. La relevancia de este problema se debe a varios factores. En primer lugar, la intensificación del proceso educativo plantea la tarea de encontrar los medios para mantener el interés de los estudiantes en el material estudiado y activar sus actividades a lo largo de toda la lección.

Los juegos educativos son un medio eficaz para resolver este problema. En segundo lugar, uno de los problemas más importantes de la enseñanza de una lengua extranjera es la enseñanza del habla oral, que crea condiciones para la divulgación de la función comunicativa de la lengua y permite acercar el proceso de aprendizaje a las condiciones del aprendizaje real, que aumenta la motivación para aprender una lengua extranjera.

Proponemos clasificar los juegos utilizados en las lecciones de lengua extranjera en dos grupos principales:

1. Juegos didácticos, que debe incluir juegos gramaticales, léxicos, fonéticos y ortográficos que contribuyan a la formación de las habilidades del habla de los estudiantes. A diferencia de los juegos en general, un juego didáctico tiene una característica esencial: un objetivo de aprendizaje claramente definido y un resultado correspondiente, que se puede fundamentar, destacar de forma explícita y se caracterizan por una orientación educativa y cognitiva. En y a través del juego didáctico, los jugadores tienen que aprender algo. 
El juego didáctico se caracteriza por lo siguiente:

- conexión con un objetivo educativo específico;

- la capacidad de repetir, interrumpir o empezar de nuevo en cualquier momento;

- apertura, es decir, el final del juego no está definido con precisión;

- adherencia a reglas explícitas que los jugadores pueden cambiar;

- satisfacción por la participación, ausencia de "consecuencias" para los jugadores (esta actividad no debe ser evaluada de ninguna manera).

La diferencia fundamental entre juegos didácticos, ejercicios y tareas es que:

Primero, el juego no tiene un patrón de comportamiento determinado, y el propio participante elige una posible versión de la interacción del habla y evalúa el resultado de su implementación. La única limitación del contenido y la forma del juego es el material educativo (tema de la lección, objetivo, resultados planificados).

En segundo lugar, el juego suele ser de naturaleza competitiva y contradictoria. Un estudiante, al entablar relaciones con compañeros en el juego, evalúa sus fortalezas no solo en comparación con otros jugadores. El juego le permite evaluar objetivamente sus capacidades.

En tercer lugar, en el juego, los escolares aprenden la comunicación interpersonal y grupal, aprenden a elegir los medios óptimos para resolver situaciones de conflicto (lingüísticas y no lingüísticas). En el juego, se forma la capacidad de correlacionar sus acciones con las acciones de otros jugadores, es decir, cooperar.

Por otra parte la experiencia recogida en esta investigación planteada para las clases de lengua extranjera, divide los juegos didácticos en dos grandes grupos: 1) "juegos con material lingüístico" y 2) "juegos en el lenguaje”.

Los primeros corresponden a clases orientadas a sistematizar el material lingüístico. Al mismo tiempo, se presta mucha atención al conocimiento de las reglas gramaticales. Por lo tanto, estos juegos son adecuados para entrenar en la etapa inicial, pero también para entrenar estructuras individuales en una etapa avanzada. Los juegos con material lingüístico pueden programarse completamente $\mathrm{y}$, por lo tanto, controlarse.

El carácter cerrado de los juegos y el protagonismo del docente se convierten en el motivo de la inadecuación de este tipo de juegos para lecciones de práctica conversacional. Pero esto no significa que no puedan aplicarse con éxito para determinados fines.

Los juegos con material lingüístico se introducen formalmente con fines educativos y sirven tanto para consolidar estructuras y reglas gramaticales como para automatizar ciertas habilidades del habla. El propósito del juego y su curso se pueden planificar fácilmente con anticipación y explicar sin ambigüedades. Son aptos para fines didácticos en actividades tradicionales. Este tipo de juegos contribuyen a la 
sistematización de material en lengua extranjera en un esquema de juego simple: bingo, lotería, juegos por parejas, juegos de mesa, crucigramas, etc. La mayoría de las veces, estos juegos se introducen para entrenar la ortografía o la sistematización del vocabulario.

Los juegos didácticos "en el idioma" se utilizan, en primer lugar, para consolidar las habilidades de escuchar, leer, hablar y escribir. Un juego comunicativo didáctico presupone tal organización de la actividad comunicativa conjunta del maestro y los estudiantes, durante la cual las características del comportamiento del habla de los escolares se manifiestan simultáneamente y se forman las habilidades de comunicación en lenguas extranjeras.

2. Los juegos de rol creativos son una de las formas de aprender idiomas extranjeros. Conceptos como el juego de roles, la simulación, el drama y la actuación a menudo se usan indistintamente, pero en realidad tienen significados diferentes.

La diferencia entre los juegos de rol y las simulaciones radica en la autenticidad de los roles desempeñados por los estudiantes. En la simulación, los estudiantes juegan su papel natural, es decir, el papel que juegan en la vida real (por ejemplo, el papel de comprador o reserva de billetes de transporte).

En el juego de roles, los estudiantes desempeñan un papel que no desempeñan en la vida real (por ejemplo, el primer ministro o la estrella de rock). El juego de rol puede considerarse como uno de los componentes o elemento de la simulación. Por lo tanto, en un juego de rol, los participantes asignan roles que se representan como parte del guión. En la simulación, la atención se centra en la interacción de un rol con otros roles, en lugar de desempeñar roles individuales. De cualquier manera, el juego de roles prepara a los estudiantes para la interacción social en un contexto social y cultural diferente.

Por lo tanto, el juego de roles es una actividad de aprendizaje muy flexible con una amplia gama de posibilidades para la diversidad y la imaginación. En los juegos de rol, se utilizan ampliamente diversas técnicas de comunicación, desarrollando así la fluidez en el lenguaje, la interacción en el aula y aumentando la motivación. El juego de roles mejora las habilidades orales de los estudiantes en cualquier situación, porque casi todo el tiempo de aprendizaje en el juego de roles se dedica a la práctica del habla, mientras que no solo el hablante, sino también el oyente es lo más activo posible, ya que debe comprender y recordar el comentario del compañero, correlacionarlo con la situación de juego, determinar qué tan relevante es para la situación y la tarea de comunicación y responder correctamente a ella. Para los estudiantes tímidos, el juego de roles ayuda al proporcionar una máscara con la que se alivian a los estudiantes con dificultades de comunicación.

A su vez, los juegos de rol se pueden clasificar de la siguiente manera: 
1. Término corto juego de rol, que es la forma más sencilla y rápida de jugar un juego que dura de 10 a 30 minutos. Puede basarse en texto o diálogo. Se puede presentar un ejemplo de este juego en forma de entrevista. Los estudiantes se dividen en parejas, después de lo cual se les dan imágenes que representan diversas situaciones problemáticas (contaminación ambiental, deforestación, falta de alimentos en los zoológicos). Uno de los estudiantes asume el rol de entrevistador, el otro el rol de entrevistado. La tarea es describir el problema y proponer una solución. El componente de juego consiste en que entre los alumnos también se designan expertos, cuya tarea es elaborar un criterio para la evaluación y posteriormente evaluar a todos los ponentes y señalar los errores cometidos. Durante este juego Existe una alta motivación y deseo de los estudiantes de mostrarse, porque uno de los criterios de evaluación puede ser el arte.

2. Completamente desarrollado o establecido Juego de roles en el que se presenta a los estudiantes una descripción de la situación y sus roles. La duración de este tipo de juegos, en promedio, toma una o dos lecciones. Como ejemplo, considere los juegos de rol verbales. Este arquetipo de juegos ocurre a través de la interacción verbal de los participantes, describiendo las acciones de sus personajes del juego, y un mentor, en cuyo rol puede ser un maestro, que describe las realidades del mundo del juego.

"Mafia" es muy popular en todo el mundo y te permite jugarla, tanto en clase como en actividades extraescolares a través de Internet. Los alumnos, prestando atención al curso del juego, comienzan a hablar espontáneamente. Su objetivo es convencer al resto de jugadores de que tal o cual participante es un mafioso / médico / sheriff. El uso de este juego en el marco de la lección ayuda a los estudiantes a desarrollar su competencia comunicativa, les enseña a defender su punto de vista, persuadirlos y los anima a tomar la iniciativa.

3. Los juegos de rol son un tipo de juego más complejo que dura una serie de lecciones o más. Al preparar juegos de rol a largo plazo, el maestro debe proporcionar a los estudiantes folletos, familiarizar a los estudiantes con el entorno del juego a través de un estudio de caso y proporcionar a los estudiantes una definición clara de la situación del juego.

En este arquetipo de juegos, los estudiantes tienen la oportunidad de crear su propio personaje de juego. Los estudiantes pueden elegir género, raza, edad, profesión, según el juego propuesto. Un ejemplo de estos juegos es la serie Dungeons and Dragons, un juego de rol de mesa. En D\&D, los estudiantes prueban el papel de héroes de fantasía con sus propias habilidades y características. El profesor asume el papel de un mentor que crea y describe una aventura en la que participan los héroes de los alumnos, establecer metas para que las logren y recompensarlas para motivarlas. El propósito de este juego es hacer que los estudiantes actúen. 
Trabajar juntos, resolviendo problemas y acertijos que el profesor les presente en el proceso de estudiar el mundo del juego. Por lo tanto, permite lograr el objetivo principal: el desarrollo de la competencia comunicativa.

Los personajes creados por los estudiantes en el transcurso de este juego se pueden utilizar en lecciones posteriores, dando a los estudiantes la oportunidad de ser quienes quieran, convirtiendo así historias monótonas sobre ellos mismos en historias fascinantes.

4. Juegos de rol por computadora. En nuestro tiempo, es difícil imaginar a una persona que no esté familiarizada con los juegos de rol de computadora. Además, en el siglo XXI, un niño pasa la mayor parte de su tiempo libre frente a la computadora. Son los juegos de computadora los que pueden motivar a un estudiante a estudiar un idioma extranjero y jugar un papel muy importante en su autodesarrollo.

Para aquellos profesores cuyos alumnos tienen acceso gratuito a Internet, los juegos de rol pueden ser una buena opción. Estos juegos brindan a los estudiantes interesados la oportunidad de establecer contacto directo con personas de todo el mundo que tienen intereses comunes pero que necesitan usar el inglés para comunicarse, destacando así el valor del aprendizaje de idiomas más allá de los grados. La mayoría de los juegos de rol de computadora brindan oportunidades para entrenar tanto las habilidades auditivas como las lectoras.

Por supuesto, es difícil usar un juego de rol de computadora para aprender gramática, pero un maestro experimentado es bastante capaz de crear un algoritmo para ejercicios de entrenamiento.

Un ejemplo de un juego de rol de computadora es Minecraft. Este juego está hecho al estilo de una caja de arena, cuyos participantes pueden construir y sobrevivir, cooperando con otros jugadores.

Los juegos de rol por computadora, con el enfoque correcto, pueden ayudar a los estudiantes a aprender no solo un idioma extranjero, sino también historia, geografía y otras materias, así como contribuir a la formación de las habilidades del estudiante.

Los juegos tienen un efecto positivo en la formación de los intereses cognitivos de los estudiantes, contribuyen al dominio consciente de una lengua extranjera. Contribuyen al desarrollo de cualidades como la independencia, la iniciativa y la capacidad de trabajar en equipo. Los estudiantes trabajan activamente, con entusiasmo, se ayudan unos a otros,

\section{Conclusiones.}

- Planificar el proceso educativo de tal manera que la carga sobre el docente no se incremente excesivamente, sino que se sienta cómodo con el nivel adecuado de salario docente, que debe estar lo más cercano posible al nivel de remuneración de la administración de las instituciones pertinentes. 
- Motivar a los alumnos para el dominio exitoso de disciplinas académicas mediante prácticas educativas, introductorias y de juegos tecnológicos en las diferentes plataformas educativas virtuales.

- La enseñanza de una segunda lengua extranjera y su estudio permitirá, en el menor tiempo posible, lograr altos resultados en el aumento de la demanda de egresados de las instituciones educativas correspondientes y su comunicación multilingüe estable.

- La enseñanza obligatoria de una segunda lengua extranjera se convertirá en una continuación razonable de la integración del individuo en el espacio mundial.

- Al usar juegos de computadora, es importante seguir las reglas que ayudan a preservar la salud y la vista de los estudiantes, y también no dejar que un buen pasatiempo los aleje de la realidad.

- La tecnología del juego ocupa un lugar importante en el proceso educativo. Se puede utilizar una amplia variedad de juegos de rol en cualquier parte del plan de estudios. Al mismo tiempo, son una herramienta muy útil que hace que el aprendizaje de una lengua extranjera sea interesante y memorable.

- Los juegos de rol brindan un estado emocional positivo a los estudiantes y una orientación comunicativa de la lección.

- La actividad de juego es la más atractiva para los escolares, por lo cual se incrementa la eficacia de la enseñanza de una lengua extranjera.

\section{Referencias bibliográficas.}

Alcedo, Y. \&. (2011). El enfoque lúdico como estrategia metodológica para promover el aprendizaje del inglés en niños de Educación Primaria. SABER.Revista Multidisciplinaria del Consejo de Investigación de la Universidad de Oriente, 23(1), 69-.

Beltrán, M. (2017). El aprendizaje del idioma inglés como lengua extranjera. . Revista Boletín Redipe, 6(4), 91-98.

Cañedo Andalia, R. (2001). Ciencia y tecnología en la sociedad: Perspectiva históricoconceptual. Acimed, 9(1), 72-76.

Celona, G. (2018). El aprendizaje eficaz: la programación neurolingüística en la enseñanza de 12 (lengua inglés). Segni e comprensione,, (93), 58-79.

Ibarra, J. F. (2019). Análisis del acceso a la formación de competencias educativas básicas para estudiantes autistas en Ecuador. . Certiuni Journal, (5), 27-37.

Merino, S. R. (2017). Técnicas de estudio para contribuir el aprendizaje desarrollador del idioma inglés en la educación superior. Espirales revista multidisciplinaria de investigación, 1(11). 
Muñoz-Repiso, A. G. (1999). El juego y las nuevas tecnologías. . Píxel-Bit. Revista de medios y educación, 89-104.

Olarte, A. C. (2016). La enseñanza del inglés en la escuela primaria: algunos problemas críticos. Colombian Applied Linguistics Journal, 18(1), 9-10.

Pérez Zúñiga, R. M. (2018). La sociedad del conocimiento y la sociedad de la información como la piedra angular en la innovación tecnológica educativa. RIDE. Revista Iberoa.

Quevedo Blánquez, L. (2016). Aprendizaje de una lengua extranjera: inglés, mediante los trabajos por proyectos en Educación Infantil.

Ribas, I. L. (2021). Calidad educativa desde la inclusión y su realidad en Ecuador. 593 . Digital Publisher CEIT, 6(1), 175-186.

Roldán Sánchez, G. A. (2016). Obstáculos en el aprendizaje del inglés como lengua extranjera en dos grupos de la población Bogotana.

Rueda Cataño, M. C. (2014). Enfoques teóricos para la adquisición de una segunda lengua desde el horizonte de la práctica educativa. Perfiles educativos, 36(143), 21-28.

Villegas, J. D.-S.-C. (2016). Variables que influyen sobre el aprendizaje del inglés como segunda lengua. Revista Internacional de Lenguas Extranjeras/International Journal of Foreign Languages, (5), 79-94.

Vivanco, V. (2001). Adquisición de Vocabulario en una Segunda Lengua. Estrategias Cognitivas-Lazos Afectivos.

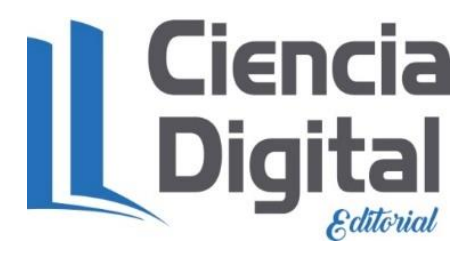




\section{PARA CITAR EL ARTÍCULO INDEXADO.}

Inca Chunata, N. M., Herrera Andrade, Z. V., Ruiz López, E. R., \& Célleri Quinde, S. P. (2021). Aspectos y problemas de aprender una segunda lengua extranjera, posibles soluciones mediante las tecnologías educativas de juegos en la enseñanza del Inglés . AlfaPublicaciones, 3(3.1), 29-41. https://doi.org/10.33262/ap.v3i3.1.74

\section{LCiencia}

El artículo que se publica es de exclusiva responsabilidad de los autores y no necesariamente reflejan el pensamiento de la Revista Alfa Publicaciones.

El artículo queda en propiedad de la revista y, por tanto, su publicación parcial y/o total en otro medio tiene que ser autorizado por el director de la Revista Alfa Publicaciones.
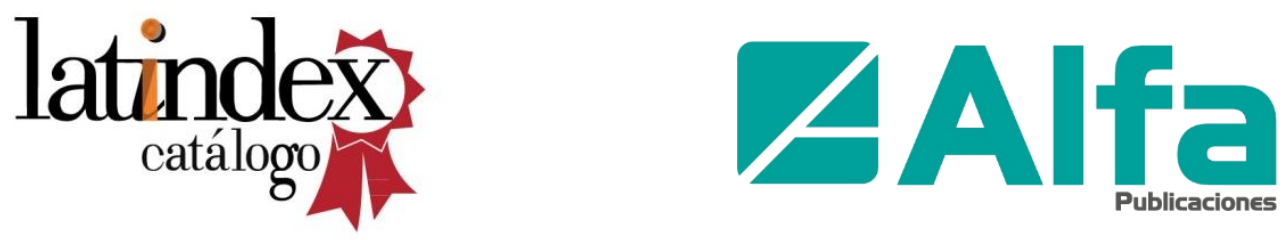